\title{
Adeno-associated virus-mediated knockdown of melanocortin-4 receptor in the paraventricular nucleus of the hypothalamus promotes high-fat diet-induced hyperphagia and obesity
}

\author{
Jacob C Garza, Chung Sub Kim, Jing Liu, Wei Zhang and Xin-Yun Lu \\ Department of Pharmacology, University of Texas Health Science Center at San Antonio, 7703 Floyd Curl Drive, San Antonio, Texas 78229, USA \\ (Correspondence should be addressed to X-Y Lu; Email: lux3@uthscsa.edu)
}

\begin{abstract}
Pharmacological and genetic studies have suggested that melanocortin-4 receptor (MC4R) signaling in the paraventricular nucleus of hypothalamus $(\mathrm{PVN})$ regulates appetite and energy balance. However, the specific role of MC4R signaling in PVN neurons in these processes remains to be further elucidated in normally developed animals. In the present study, we employed RNA interference to determine whether MC4R knockdown in the PVN modulates food intake and body weight in adult rats. Adeno-associated viral (AAV) vectors encoding short hairpin RNAs targeting MC4R (AAV-shRNA-MC4R) were generated to induce MC4R knockdown in the PVN. By in situ hybridization, we
\end{abstract}

detected a high-level expression of Dicer, a key enzyme required for shRNA-mediated gene silencing, along the entire rostrocaudal extent of the PVN. Bilateral injection of AAV-shRNA-MC4R vectors into the PVN of the adult rat resulted in significant and specific reduction of MC4R mRNA expression. Animals with MC4R knockdown exhibited an increase in food intake and excessive body weight gain when exposed to a high-fat diet. Our results provide evidence that $A A V$-mediated silencing of MC4R on PVN neurons promotes hyperphagia and obesity in response to the dietary challenge in the adult animal.

Journal of Endocrinology (2008) 197, 471-482

\section{Introduction}

Melanocortin-4 receptor (MC4R) in the central nervous system (CNS) plays a critical role in the control of energy balance. Activation of MC4R by $\alpha-\mathrm{MSH}$, a derivative of proopiomelanocortin (POMC), decreases food intake and increases energy expenditure. Conversely, inhibition of MC4R by agouti-related protein (AgRP) increases food intake and decreases energy expenditure. MC4R deficiency in mice or humans results in hyperphagia and a severe obesity syndrome (Huszar et al. 1997, Yeo et al. 1998, Vaisse et al. 2000).

MC4R is broadly expressed in many brain areas implicated in the regulation of energy balance (Mountjoy et al. 1994, Kim et al. 2000, Kishi et al. 2003, Lu et al. 2003, Kas et al. 2004). One of the brain regions that highly express MC4R is the paraventricular nucleus of the hypothalamus (PVN) (Mountjoy et al. 1994, Kishi et al. 2003, Lu et al. 2003). Studies suggest that the PVN is one of the key neuroanatomical substrates in mediating MC4R signaling in the regulation of energy homeostasis. Lesions of the PVN result in hyperphagia in rats and mice (Weingarten et al. 1985, Sawchenko 1988,1998). Microinjection of MC4R agonists MTII or $\boldsymbol{\alpha}$-MSH into the PVN inhibits food intake, whereas microinjection of MC4R antagonists AgRP, SHU9119 or HS014, into this region stimulates food intake (Cowley et al.
1999, Kask et al. 2000, Kim et al. 2000, Wirth \& Giraudo 2001). It has also been demonstrated that overexpression of MC4R antagonist agouti in the PVN using adeno-associated virus (AAV) causes increased food intake and body weight (Kas et al. 2004). These pharmacological data support the importance of MC4R activity in the PVN in the control of appetite and body weight. However, the effects of MC4R ligands in these pharmacological studies are not necessarily derived from interaction with MC4R on local PVN neurons but may also involve binding to MC4R on afferent terminals. The PVN receives afferent inputs from many brain areas containing MC4R-expressing neurons such as the bed nucleus of terminals and nucleus of solitary tract (Sawchenko \& Swanson 1983, Shioda et al. 1990). Therefore, the specific role of MC4R in PVN neuronal populations remains to be further elucidated.

Using the MC4R null mouse model in which MC4R is reactivated by Cre-recombinase, Balthasar et al. (2005) demonstrate that restoration of MC4R in neurons located primarily in the PVN, nucleus of the lateral olfactory tract, and medial amygdala can completely reverse increased food intake without affecting energy expenditure. Additionally, they show that reactivating MC4R specifically in the PVN in the adult MC4R-null mice causes a mild reduction of body weight (Balthasar et al. 2005). These findings suggest that MC4R on 
PVN neurons plays a critical role in maintaining energy homeostasis when MC4R is genetically absent from other brain regions and tissues. However, it is not known whether disruption of MC4R on PVN neurons in normally developed wild-type animals is sufficient to alter feeding and weight gain. In the present study, we used AAV vector-mediated RNA interference (RNAi) to suppress endogenous MC4R expression specifically in the PVN of the adult rat. Our results show that MC4R knockdown in PVN neurons increases food intake and body weight gain in response to a high-fat diet, suggesting that MC4R signaling in PVN neurons may protect against overeating and obesity under the dietary challenge.

\section{Materials and Methods}

\section{Design and construction of short hairpin RNAs (shRNAs)}

To identify effective targeting sequences for RNAi, the fulllength coding sequence of rat MC4R was analyzed and a list of ten candidate 21 base oligonucleotides was generated. Homology analyses were conducted for each oligonucleotide using the rat genome database to ensure that the sequence is unique to the rat MC4R gene. Three 21 base oligonucleotides were chosen to design shRNAs. In addition, a 21 base scrambled oligonucleotide sequence was used for specificity control. To construct shRNAs, forward oligonucleotides were designed that contained the 21 base sense and antisense sequences connected with a hairpin loop (TTCAAGAGA) followed by a poly(T) termination signal. Reverse oligonucleotides complementary to the forward sequence were also generated. BamHI cleavage site overhangs (gatcc) were added to the $5^{\prime}$-end of each forward oligonucleotide and EcoRI cleavage overhangs (attc) were added to the $5^{\prime}$-end of each reverse oligonucleotide for ligation into the pSIREN-DNR mammalian expression vector (Clontech). Additionally, a MLUIsite (ACGCGT) was added to the $3^{\prime}$-end of the forward and $5^{\prime}$-end of the reverse oligonucleuotides for cloning into the pTR-UF5 AAV vector. The corresponding forward and reverse oligonucleotides were annealed to generate a double-stranded DNA construct that was ligated into the pSIREN-DNR expression vector of the production of shRNAs (illustrated in Fig. 1A). The following constructs were selected for targeting the rat MC4R mRNA:

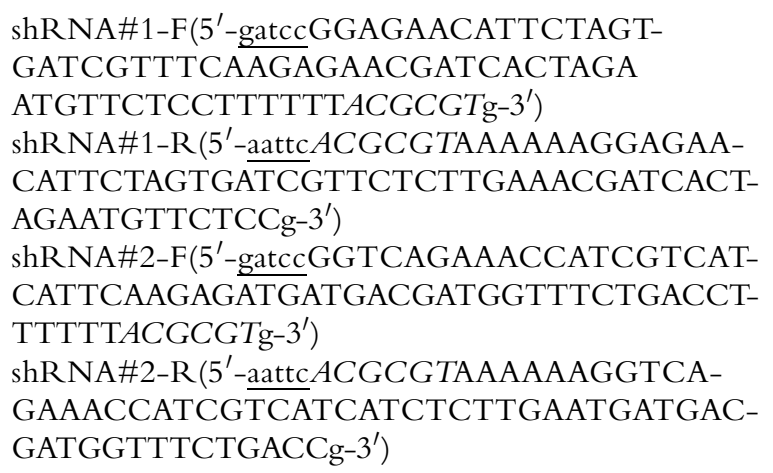

shRNA\#3-F(5'-gatccACCTTCAAAGAGATCATCTGTTTCAAGAGAACAGATGATCTCTTTGAAGGTTTTTTTACGCGTg-3')

shRNA\#3-R ( $5^{\prime}$-aattc $A C G C G T A A A A A A A C C T T-$ CAAAGAGATCATCTGTTCTCTTGAAACAGATGATCTCTTTGAAGGTg-3')

The expression of shRNAs were driven by the human RNA polymerase III-dependent U6 promoter as we described previously (Paz et al. 2007).

Screening of candidate $\operatorname{sh} R$ As for MC4R silencing in vitro

To determine the efficacy of the selected shRNAs to suppress MC4R expression, the full-length cDNA of the rat MC4R (from a plasmid originally obtained from Dr Ira Gantz, University of Michigan) was cloned into the $\mathrm{pEGFP-C1}$ vector in which the CDNA of MC4R was fused to the C-terminal of EGFP for fluorescent quantification of MC4R expression. The ability of individual shRNAs to silence the expression of the rat MC4R was examined in the HEK-293 cell line. The pEGFPC1 vector expressing the rat MC4R and the pSIREN vector expressing shRNAs that target MC4R or scrambled control shRNA were co-transfected into HEK-293 cells. The expression of MC4R was examined 24 and $48 \mathrm{~h}$ after co-transfection. As a specific MC4R antibody is not available, the expression level of fused EGFP was used as an index of MC4R expression. Moreover, to determine the specificity of shRNAs in silencing MC4R, the pSIREN vector expressing MC4R-shRNA was co-transfected with an expression vector carrying a gene sharing no sequence homology with MC4R, i.e., the agouti-related peptide (AgRP) gene. The rat AgRP cDNA sequence was amplified by PCR and cloned into the pEGFP-C1 vector (Clontech) as described previously for MC4R-EGFP. The pEGFP-AgRP vector and the pSIREN vector expressing the MC4R-shRNA were co-transfected into HEK-293 cells. The expression of AgRP was examined $24 \mathrm{~h}$ after co-transfection.

\section{Production and purification of adeno-associated virus expressing $\operatorname{sh} R N A s$}

To investigate long-term effect of MC4R gene silencing on energy balance, the shRNA constructs were then cloned into an adeno-associated virus, serotype 2 (AAV2) for stable siRNA delivery. The pSIREN vector expressing shRNAs were digested using MluI to release a 460 bp fragment containing the shRNA sequence. The pTR-UF5 vector, an AAV-inverted terminal repeat-containing vector that encodes EGFP, was linearized using MluI. The fragment that was released from the pSIREN vector was ligated into the pTR-UF5 vector using T4 DNA ligase, transformed into DH10B Escherichia coli bacteria cells, and grown on ampicillin selective plates. Colonies were selected and grown in LB ampicillin $(50 \mu \mathrm{g} / \mathrm{ml})$ after which the plasmid DNA was purified and 
A

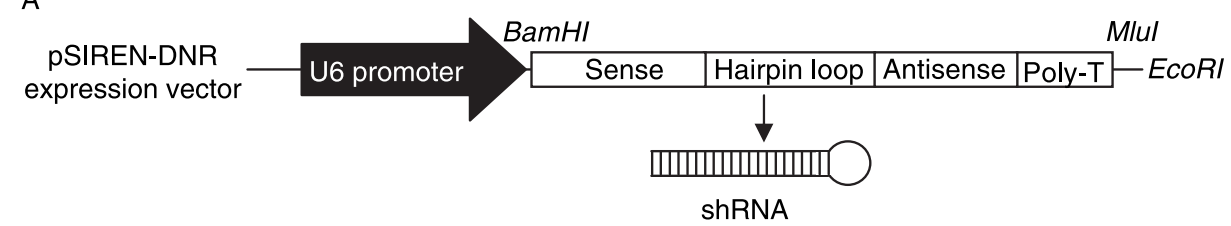

B

Control-shRNA

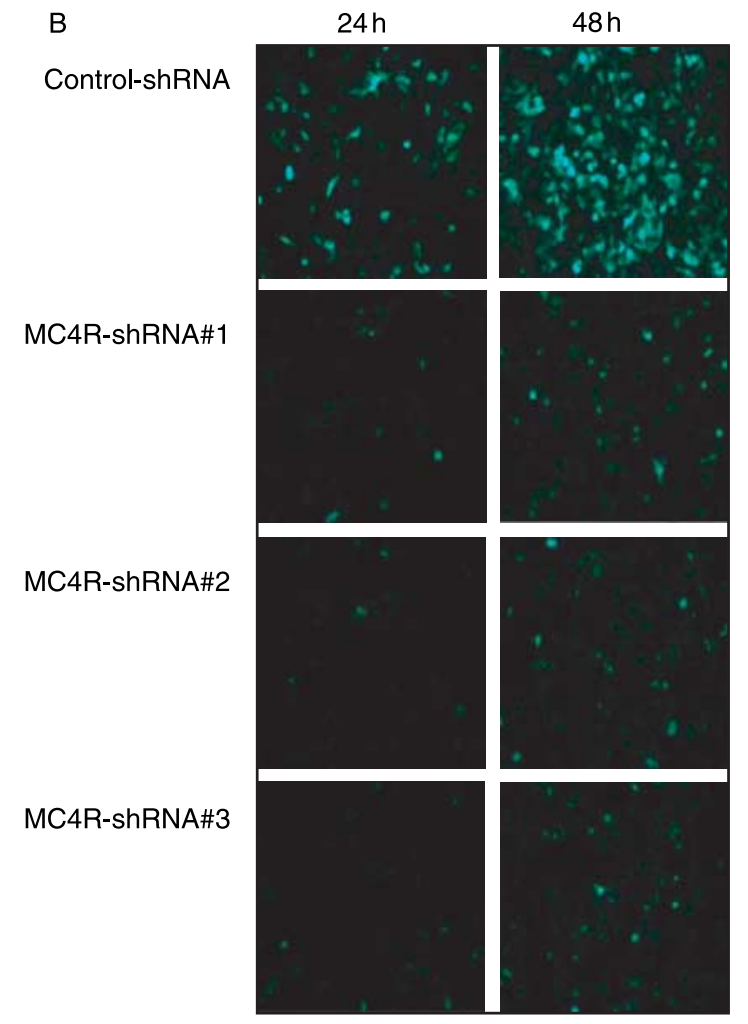

$48 \mathrm{~h}$

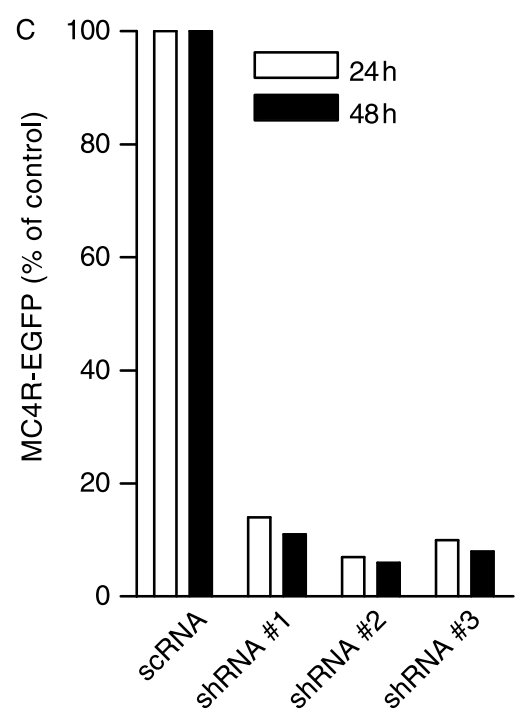

Figure 1 Inhibition of rat MC4R-EGFP fusion protein by specific shRNAs. (A) Schematic of shRNA expression vector. The oligonucleotides for each shRNA were cloned into the pSIREN-DNR expression vector using BamHI and EcoRI. (B) The vector encoding the rat MC4R-EGFP fusion protein (pEGFPcl-rMC4R) was introduced into HEK-293 cells with an RNAi vector expressing control RNA (pSIREN-control-shRNA) or shRNAs against the rat MC4R gene (pSIREN-MC4R-shRNA). The fluorescent images were taken at 24 and $48 \mathrm{~h}$ after co-transfection showing the intensity of fluorescence and cells expressing MC4R-fusioned protein. (C) Quantitative analysis showing percentage of inhibition of MC4R-EGFP expression by shRNAs.

screened for the presence of the insert. Positive plasmids were used to generate AAV expressing shRNAs.

For AAV purification, HEK-293 cells were grown until confluent on $150 \mathrm{~cm}$ cell culture plates and subsequently transfected via the calcium phosphate transfection protocol using a 3:1 ratio of shRNA in pTR-UF5 vector to $\mathrm{pDC}_{2}$ helper plasmid. After transfection, the cells were incubated in DMEM (10\% BSA) at $37^{\circ} \mathrm{C}$ with $5 \% \mathrm{CO}_{2}$ for $48 \mathrm{~h}$ after which they were harvested. AAV was purified using a protocol adapted from Xie et al. (2004). The collected cells were centrifuged at $4050 \mathrm{~g}$ for $2 \mathrm{~h}$ at $4{ }^{\circ} \mathrm{C}$ to collect the cell pellet and were resuspended in TD buffer $(140 \mathrm{mM} \mathrm{NaCl}, 5 \mathrm{mM}$ $\mathrm{KCl}, 0.7 \mathrm{mM} \mathrm{K}_{2} \mathrm{HPO}_{4}, 25 \mathrm{mM}$ Tris-HCL (pH 7-4)). Trypsin $(0 \cdot 0125 \%)$ and sodium deoxycholate $(0.5 \%)$ were added for
$30 \mathrm{~min}$ at $37^{\circ} \mathrm{C}$ to lyse the cells. Next, the lysed cell solution was homogenized using a Dounce homogenizer and a graded series of syringe needles (18G, 20G, and 23G). Briefly, the lysate was centrifuged in a table top centrifuge $(1000 \mathrm{~g}$ for $10 \mathrm{~min}$ ) to remove the large cellular debris. Cesium chloride was added to the lysate to achieve a concentration of $3.3 \mathrm{M}$ with a refractive index of $1 \cdot 37$. The samples were centrifuged in a hanging basket rotor at $178305 \mathrm{~g}$ for $24 \mathrm{~h}$. Fractions were collected from the cesium chloride gradient that correspond to a refractive index of $1 \cdot 35-1 \cdot 40$, which is the density range expected for AAV. Lipids and other debris were removed from the top of the tube and discarded. Similar fractions were pooled; and this step was repeated twice, but allowed to centrifuge for $60 \mathrm{~h}$ on the second repeat. Once the fractions 
were collected, those samples corresponding to a refractive index of 1.37 were dialyzed overnight in fresh DMEM and then concentrated using Nanosep 10K Omega centrifugation column (Pall Life Sciences, East Hills, NY, USA). Serial tenfold dilutions of the vector were used to co-infect 293 cells with adenovirus type 5 . Twenty-four hours later, the number of EGFP-expressing cells in the infected cells will be counted and the titer of the vector was calculated.

To test the efficacy of MC4R-shRNA in AAV vectors, we created a vector expressing MC4R-DsRed (a red fluorescent protein) fusion protein. MC4R was cloned into the pDsRedExpress-C1, a mammalian expression vector that encodes DsRed-Express, a variant of red fluorescent protein (Clontech). DsRed-Express has improved solubility of the protein and reduced time from transfection to detection of red fluorescence. The constructed pDsRed-Express-C1-rMC4R vector was then co-transfected with $A A V$ vectors expressing shRNAs into HEK-293 cells. The expression of DsRed was used as an indicator of MC4R expression. EGFP was used as a marker for shRNA expression.

\section{Expression of Dicer in the brain}

Stable RNAi that is achieved by AAV-mediated in vivo transcription of shRNAs requires the presence of Dicer, an enzyme for shRNA processing into functional small interference RNA (siRNA). To determine the expression of Dicer mRNA in the rat brain, a $668 \mathrm{bp}$ cRNA probe was designed against the rat Dicer1 cDNA. Two primers (Dicer 1 Forward $5^{\prime}$-gcgcggtaccTCTTTCATCTCACCTGTAACT-3' and Dicer 1 Reverse $5^{\prime}$-gcgcggatccGTGATAGCGTGCTTTAAGAAG- $3^{\prime}$ ) were used to amplify the fragment from nucleotide 3550 to 4218 of the full-length cDNA. In order to facilitate cloning into a plasmid, restriction enzyme sites were added to the $5^{\prime}$-end of each primer. A KpnI cut site (ggtacc) was added to the $5^{\prime}$-end of the forward primer and a $\mathrm{BamHI}$ cut site (ggatcc) was placed at the $5^{\prime}$-end of the reverse primer. The amplified fragment was subsequently cloned into the pBK-CMV vector (Stratagene, La Jolla, CA, USA) and used for in vitro transcription. Purified plasmid DNA containing the probe against the rat Dicer 1 was linearized with KpnI for synthesizing an antisense cRNA probe and transcribed with T3 RNA polymerase or linearized with BamHI for synthesizing a sense cRNA probe and transcribed with T7 RNA polymerase. Dicer mRNA distribution was determined by in situ hybridization as described below.

\section{In vivo delivery of $A A V$ vectors expressing shRNAs}

Adult male Sprague-Dawley rats (Charles River Laboratories, Wilmington, MA, USA) were housed in groups before surgery and maintained on a 12-h light:12-h darkness cycle with ad libitum access to water and food. The procedures were conducted in accordance with the Guide for the Care and Use of Laboratory Animals.
AAV vectors expressing MC4R-shRNAs were injected bilaterally into the PVN at a $10^{\circ}$ angle using the following coordinates measured from bregma: AP $-1.7 \mathrm{~mm}, \mathrm{ML} \pm$ $1.8 \mathrm{~mm}$, DV $-7 \cdot 3 \mathrm{~mm}$ from dura (Paxinos \& Watson 1998). One microliter of AAV-MC4R-shRNA $\left(2 \times 10^{9}\right.$ infectious particles $/ \mathrm{ml}$ ) was infused over a $15-\mathrm{min}$ period. AAV-controlshRNA $\left(2 \times 10^{9}\right.$ infectious particles $\left./ \mathrm{ml}\right)$ were injected into the PVN and used as specificity controls. Following injection, the injector was kept in place for additional $5 \mathrm{~min}$ to allow for diffusion and prevention of backflow through the needle track before the injector was withdrawn. After surgery, the rats were housed individually. The rats were provided with regular rodent chow (containing $19 \cdot 0 \%$ crude protein, $5 \cdot 0 \%$ fat, and $5 \cdot 0 \%$ crude fiber; Harlan Teklad, Madison, WI, USA) for 14 days and then switched to a high-fat diet (containing $20 \cdot 0 \%$ protein, $45.0 \%$ fat, and 35.0\% carbohydrate; Research Diet, New Brunswick, NJ, USA) for 21 days. Food intake and body weight were measured every 2 days. Upon completion of the experiment the rats were rapidly decapitated and their brains were frozen in a dry-ice/isopentane bath, sectioned at $20 \mu \mathrm{m}$ in a cryostat, and mounted onto poly-lysine-coated glass slides. Slides were stored at $-80{ }^{\circ} \mathrm{C}$ until they were processed for in situ hybridization.

The injection site was confirmed by visualizing GFP fluorescence using a fluorescent microscopy. The rats with injection sites outside the PVN were excluded from the data analyses of feeding, body weight, and mRNA expression.

\section{In situ hybridization}

Antisense cRNA probes directed against the sequences of the rat Dicer, MC4R, and arginine vasopressin (aVP) mRNAs were labeled with ${ }^{35} \mathrm{~S}-\mathrm{UTP}$ and ${ }^{35} \mathrm{~S}-\mathrm{CTP}$ using the standard transcription system as described in our previous studies $(\mathrm{Lu}$ et al. 2002, 2003). Briefly, the brain sections mounted on polylysine-coated slides were fixed in $4 \%$ paraformaldehyde for $1 \mathrm{~h}$ and rinsed in $2 \times \mathrm{SSC}(300 \mathrm{mM} \mathrm{NaCl}, 30 \mathrm{mM} \mathrm{Na}$ citrate $(\mathrm{pH} 7 \cdot 2))$ three times for $5 \mathrm{~min}$. Tissue slides were then acetylated in $0 \cdot 1 \mathrm{M}$ triethanolamine ( $\mathrm{pH} 8 \cdot 0$ ) with $0 \cdot 25 \%$ acetic anhydride (for $10 \mathrm{~min}$ ) and dehydrated through a graded series of alcohol (50-100\%). Hybridization was accomplished by the addition of $70 \mu \mathrm{l}$ of the diluted probe to the slide followed by incubation at $55^{\circ} \mathrm{C}$ overnight. During the incubation, the slides were placed over filter paper saturated with $50 \%$ formamide in a tightly sealed container. The slides were then rinsed in $2 \times$ SSC and incubated in RNase A buffer $(200 \mu \mathrm{g} / \mathrm{ml})$ for $1 \mathrm{~h}$ at $37^{\circ} \mathrm{C}$ followed by a series of washes of increasing stringency $(2 \times, 1 \times$, $0.5 \times \mathrm{SSC}$, for $5 \mathrm{~min}$ each at room temperature). Finally, the slides were placed in $0.1 \times$ SSC at $70{ }^{\circ} \mathrm{C}$ for $1 \mathrm{~h}$, rinsed in distilled water, and dehydrated in a graded series of alcohol. The slides were exposed to $\mathrm{X}$-ray film. The sections were exposed to $\mathrm{X}$-ray films for $3 \mathrm{~h}$ (aVP), 14 days (MC4R), or 5 days (Dicer). Signal specificity was ensured either by hybridization with sense-strand probes or pretreatment of brain sections with RNase A $\left(200 \mu \mathrm{g} / \mathrm{ml}\right.$ at $37^{\circ} \mathrm{C}$ for $\left.60 \mathrm{~min}\right)$. 


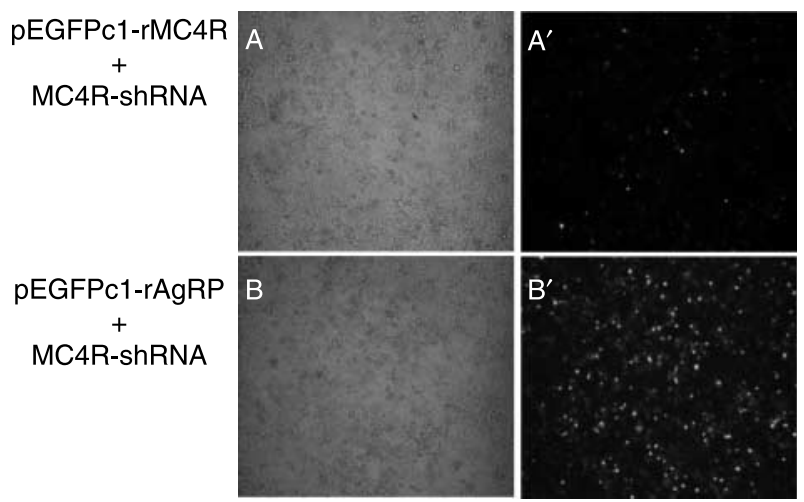

Figure 2 Specificity of gene silencing. (A and $B$ ) Bright field and $\left(A^{\prime}\right.$ and $B^{\prime}$ ) fluorescent field. (A and $A^{\prime}$ ) Co-transfection of the rMC4R-EGFP expression vector with a vector expressing shRNA for MC4R. (B and B') Co-transfection of the rAgRP-EGFPexpression vector with a vector expressing shRNA for MC4R. MC4R-shRNA suppresses rMC4R-EGFP expression but has no effect on rAgRP-EGFP expression.

\section{Semi-quantitative $m R N A$ analysis}

The levels of MC4R and aVP mRNA were analyzed by computer-assisted optical densitometry. Digital images of the brain sections were captured from $\mathrm{X}$-ray films in the linear range of the gray levels using a CCD camera (Model XC-77; Sony, Tokyo, Japan). The integrated optical density of in situ hybridization signals was determined using an MCID system (Imaging Research, Inc., St. Catharines Ontario, Canada). A standard template for sampling was employed.Signals were expressed as optical density levels above threshold. The threshold level is defined as 3.5 s.D.s above the mean optical density of the background. Five brain sections throughout the rostrocaudal extent of the PVN from each animal that were anatomically matched were used for quantification of MC4R and aVP mRNA levels. Four brain sections containing the dorsomedial hypothalamus (DMH), amygdala, and dorsal striatum from each animal were used for quantification of MC4R mRNA levels. Equivalent planes of coronal brain sections were verified using the Nissl-stained adjacent slides for analysis between animals.

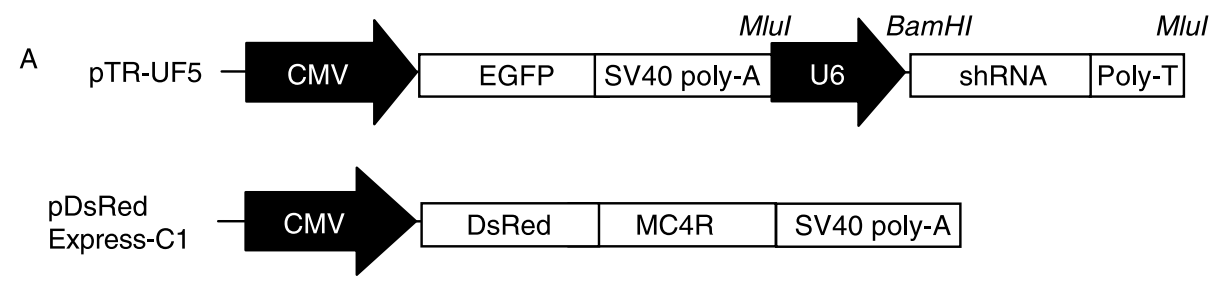

B
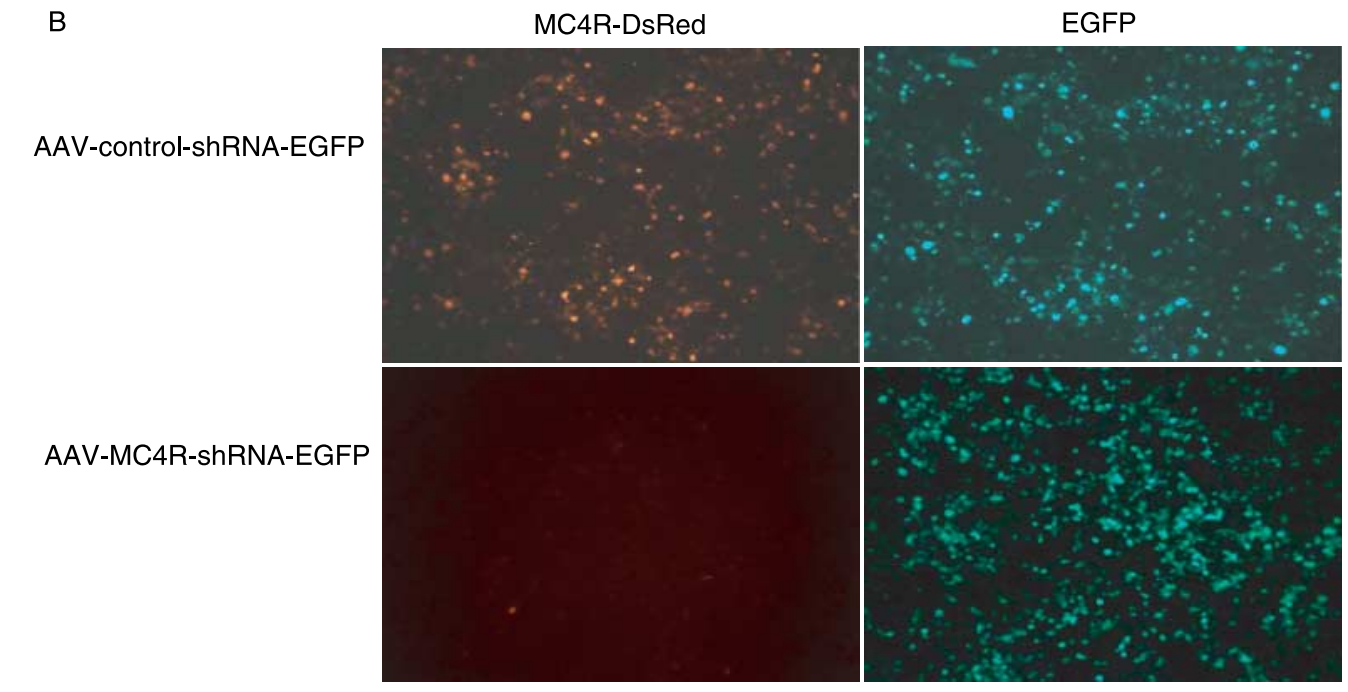

Figure 3 AAV-mediated knockdown of MC4R expression in HEK-293 cells. (A) Schematic of the construct of the AAV vector. Top, AAV vector pTR-UF5 containing EGFP under the control of the CMV promoter and shRNA driven by the U6 promoter. Bottom, pDsRed-Express-C1 vector expressing MC4R tagged with DsRed (RFP). (B) Inhibition of the expression of MC4R-DsRedfusion protein by AAV-MC4R-shRNA-EGFP. Fluorescent images in DsRed and GFP - $48 \mathrm{~h}$ after co-transfection with MC4R-DsRed with either AAV-control-shRNA-EGFP or AAV-MC4R-shRNA-EGFP. 
Image processing

Autoradiogram images were captured with a CCD camera using a MCID system. Bright-, dark- and fluorescent-field images were captured on a DP controller digital camera attached to a $\mathrm{B} \times 52$ Olympus microscope. Images were processed using Adobe Photoshop software (Adobe Systems Inc).

\section{Statistical analysis}

Data were expressed as mean \pm s.E.M. One-way ANOVA with repeated measures was used to analyze the food intake and body weight gain data. Student's $t$-test was performed for mRNA expression data analyses. $P<0.05$ was considered statistically significant.

\section{Results}

Screening shRNAs for efficient silencing of $M C 4 R$ in vitro

To determine the efficiency and specificity of the candidate MC4R shRNAs, we generated three shRNA vectors that target different regions of the rat MC4R mRNA (MC4RshRNAs). A scrambled control vector was also generated that is not complementary to any known mammalian gene sequence. These vectors were transfected into HEK-293 cells together with a plasmid encoding rat MC4R fused with EGFP and the ability of the shRNAs to specifically suppress MC4R expression was examined by immunofluorescence. We found that co-transfection of the shRNA but not the scrambled control vectors greatly suppressed the expression of MC4REGFP, as indicated by decreased number of cells with green fluoresence (Fig. 1B). Quantative analyses indicated that the extent of reduction of MC4R-EGFP induced by three MC4R-shRNAs varied ranging from 86 to $94 \%$ (Fig. 1C). The MC4R-shRNA\#2 with the highest efficacy (94\% of inhibition) was used for the subsequent experiments.

To determine the specificity of shRNAs in suppressing the rat MC4R gene, the vector expressing MC4R-shRNA (referring shRNA\#2 in the following in vitro and in vivo experiments) was then co-transfected with a vector expressing an unrelated gene, AgRP, which is fused with EGFP for fluorecent detection. It was revealed that MC4R-shRNA specifically inhibited MC4R expression, but not AgRP expression (Fig. 2).

\section{$A A V$-mediated silencing of $M C 4 R$ in vitro}

To facilitate the delivery of shRNA constructs to neurons and to induce long-term expression of shRNAs for evaluation of chronic effects of MC4R knockdown on energy balance, we used AAV vectors to deliver shRNAs to a single brain area. In order to confirm that the MC4R-shRNA was delivered to the desired brain region and to monitor the transduction efficiency of AAV, we generated a AAV vector containing two transcription units, one encoding shRNA under the control of the U6 promoter and another encoding EGFP under the control of the cytomegalovirus (CMV) promoter (Fig. 3A). Expression of EGFP was used as an index of effective transduction of the AAV vector and detection of deposition site following stereotaxic injection. To confirm the efficacy of MC4R-shRNA in AAV vectors prior to the use in vivo, the vector encoding MC4R fused with DsRed for detection of MC4R expression was co-transfected with either the AAVMC4R-shRNA-EGFP or AAV-control-shRNA-EGFP vector into HEK-293 cells. As shown in Fig. 3B, AAV-MC4RshRNA-EGFP completely suppressed the expression of MC4R in comparison with the cells treated with AAVcontrol-shRNA-EGFP. The robust EGFP expression in AAV-shRNA-transfected cells and healthy propagation of cells indicated that shRNAs were not toxic to the cells.

\section{Dicer $m R N A$ expression in the brain}

To determine the expression pattern of Dicer in the brain, we constructed a vector expressing a 668 base fragment of the rat Dicer cDNA which was produced by RT-PCR, and generated sense and antisense cRNA probes for detection using in situ hybridization. Dicer mRNA was selectively expressed in various rat brain areas with high levels of expression in the hippocampus, habenula, and hypothalamus (Fig. $4 \mathrm{~A}^{\prime}$ and $\mathrm{B}^{\prime}$ ). Within the hypothalamus, high levels of Dicer mRNA were observed in the PVN, ventromedial, dorsomedial, and arcuate nuclei. Particularly, high levels of hybridization signals for Dicer mRNA were detected throughout the rostrocaudal extent of the PVN, where MC4R-expressing neurons are located (Fig. 5B).

\section{Effect of $A A V$-mediated silencing of $M C 4 R$ in the rat brain on food} intake and body weight

First, the specificity of MC4R in situ hybridization signals was determined by hybridizing adjacent brain sections with sense and antisense cRNA probes. While the sense probe showed only background noise, the antisense probe revealed specific in situ hybridization signals in the PVN and striatum as shown in Fig. 5. To test the efficiency of AAV-mediated MC4R gene knockdown, AAV vectors expressing control shRNA or MC4R-shRNA were stereotaxically delivered to each side of the PVN. AAV-mediated EGFP expression was restricted to the deposition sites (Fig. 6A and B), indicating very limited diffusion of AAV vectors in the brain. In situ hybridization demonstrated that the side of the PVN infected by AAVMC4R-shRNA exhibited reduction of MC4R mRNA expression (Fig. 6C). Nissl staining revealed no cell loss or abnormalities in cell density and cytoarchitecture in the PVN, suggesting no cellular toxicity (Fig. 6D).

To determine the effects of MC4R knockdown in the PVN on feeding and body weight, the rats received bilateral injection of AAV-control-shRNA or AAV-MC4R-shRNA vectors into the PVN. After microinjection, animals were provided with a regular chow diet for 14 days. Food intake and body weight gain 

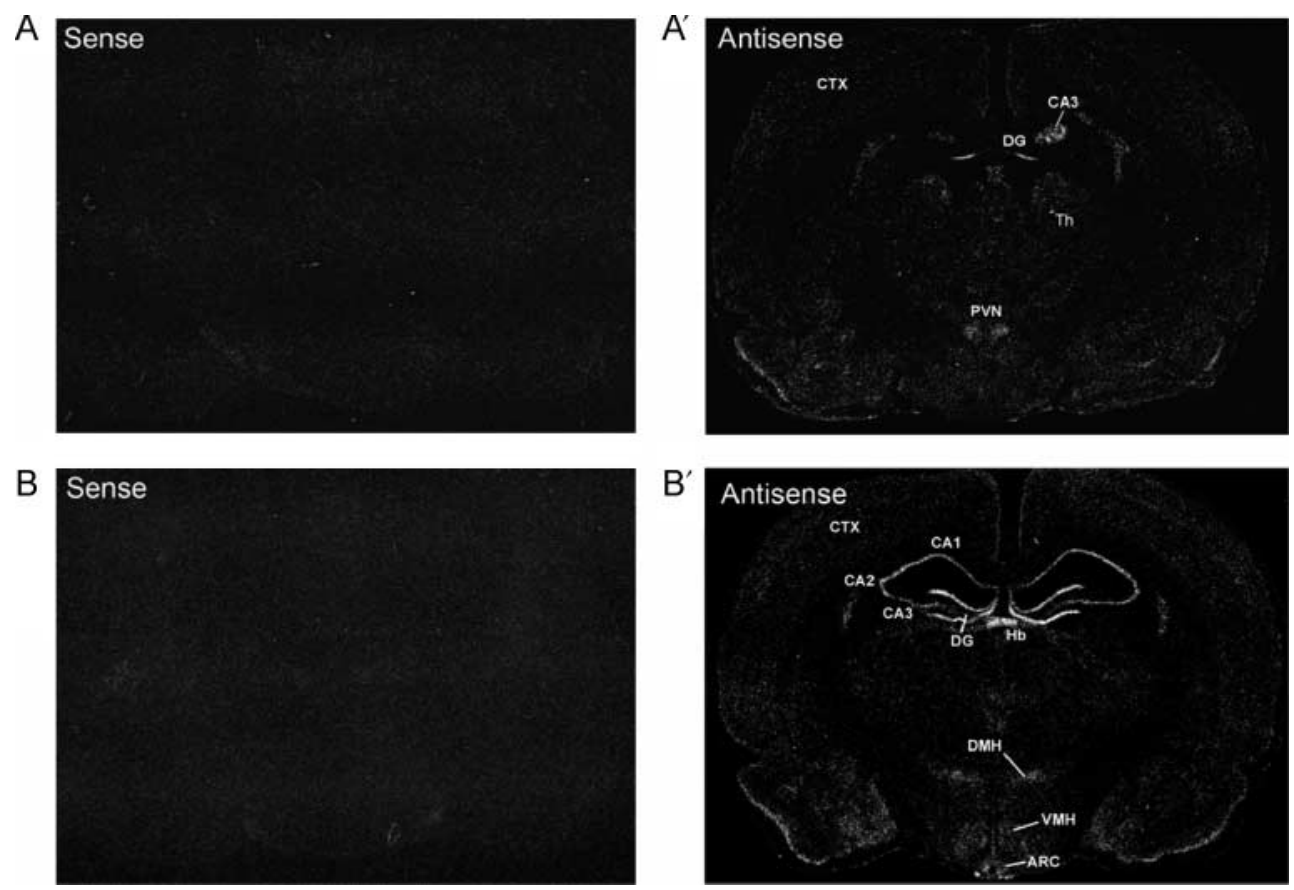

Figure 4 Dicer mRNA expression. Images of dark-field autoradiograms showing Dicer mRNA expression in the rat brain. (A and B) Adjacent brain sections at the coronal planes of the hypothalamus were used for in situ hybridization with ${ }^{35} S$-labeled sense and antisense $\left(A^{\prime}\right.$ and $\left.B^{\prime}\right)$ Dicer $c R N A$ probes. ( $A$ and $A^{\prime}$ ) Rostral level and (B and $\mathrm{B}^{\prime}$ ) caudal level. ARC, arcuate nucleus; $\mathrm{CA} 1-3$, area 1-3 Ammon's horn; CTX, cortex; DG, dentate gyrus; DMH, dorsomedial hypothalamus; Hb, habenula; PVN, paraventricular nucleus of hypothalamus; $\mathrm{TH}$, thalamus; $\mathrm{VMH}$, ventromedial hypothalamus.

showed no significant difference between two treatment groups (food intake: $F(1,96)=0 \cdot 135 ; P=0.7229$; body weight: $F$ $(1,104)=0 \cdot 02 ; P=0 \cdot 886$; Fig. 7A). Subsequently, a high-fat diet was provided to the rats for 21 days. Food intake and body weight were measured every 2 days until rats were killed. Rats treated with AAV-MC4R-shRNA exhibited significant increase in both food intake and body weight gain in response to a high-fat diet (food intake: $F(1,72)=5 \cdot 512 ; P=0 \cdot 0435$; body weight: $\mathrm{F}(1,72)=5 \cdot 448 ; P=0 \cdot 0445$; Fig. $7 \mathrm{~B})$. The rats with no detectable EGFP in the PVN or with EGFP outside the PVN (representing missed or failed injection) were excluded from the feeding and body weight analyses.
To determine the extent of suppression of MC4R mRNA by AAV-mediated RNAi, the level of MC4R mRNA in the PVN was measured at the end of the experiment using in situ hybridization. The levels of MC4R mRNA were significantly decreased in the PVN by AAV-MC4R-shRNA vectors compared with AAV-control-shRNA control (Fig. 8A and B). To determine the specificity of MC4R-shRNA in the PVN, adjacent brain sections were used to evaluate the expression level of a gene unrelated to MC4R, aVP, in the PVN. No significant changes in aVP mRNA expression were induced by AAV-MC4R-shRNA vectors (Fig. 8C). In addition, MC4R knockdown was specific to the microinjection site, as MC4R
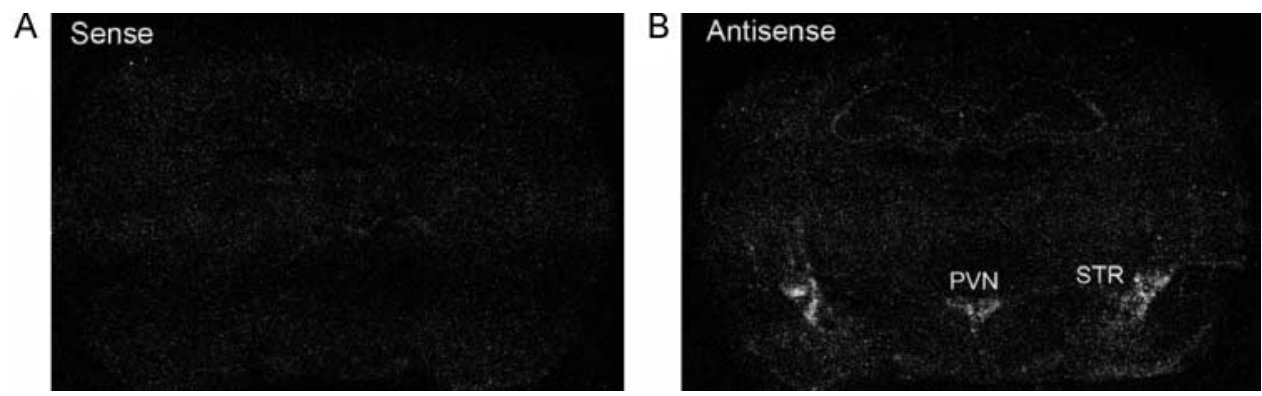

Figure 5 Specificity of MC4R mRNA expression. Adjacent brain sections were hybridized with ${ }^{35}$ S-labeled sense and antisense MC4R cRNA probes. Dark-field in situ hybridization autoradiograms showing nonspecific background ( $A$, sense probe) and specific hybridization signals ( $B$, antisense probe). 

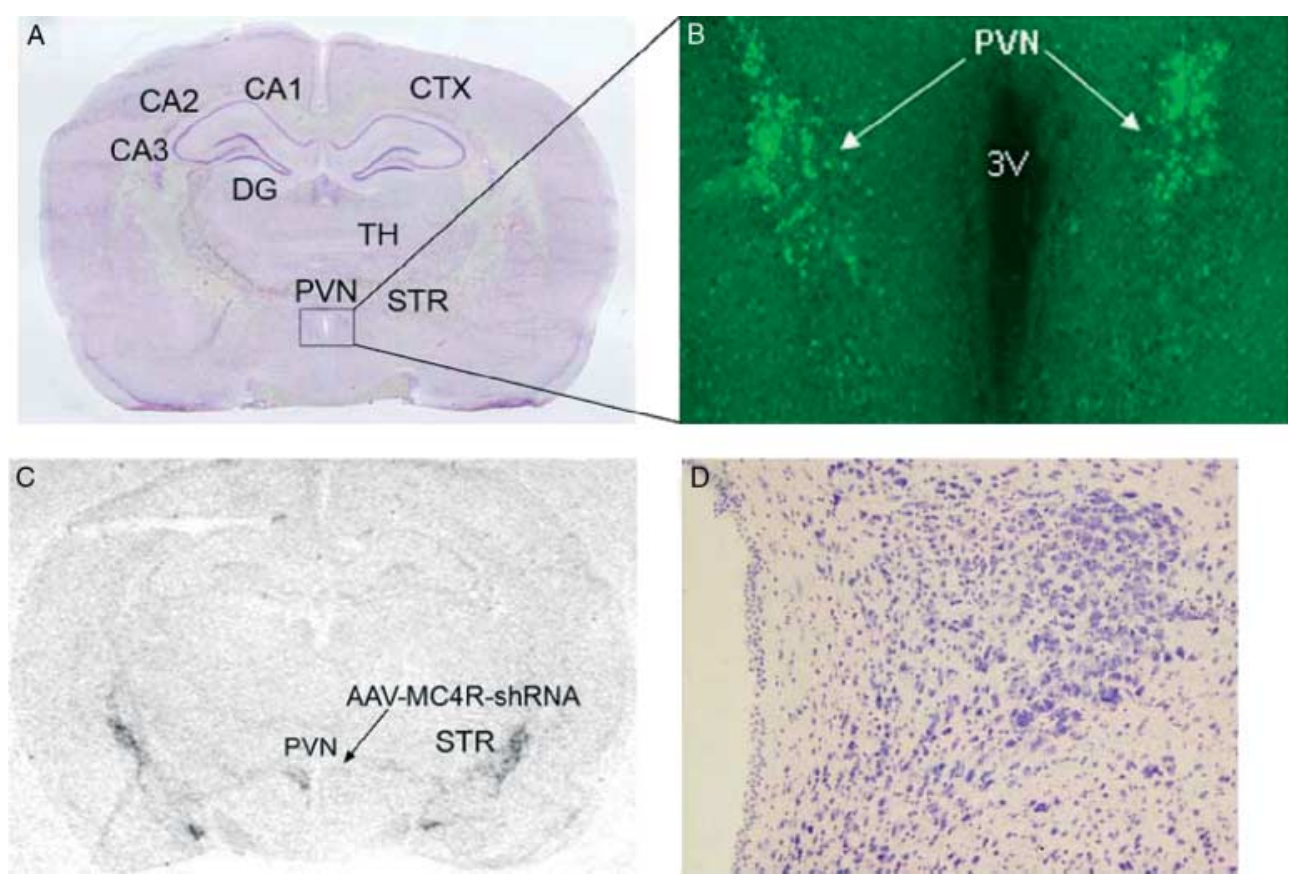

Figure 6 AAV-mediated MC4R knockdown in the paraventricular nucleus of the hypothalamus. AAV-controlshRNA and AAV-MC4R-shRNA vectors were stereotaxically delivered into one side of the PVN of the rat. The animal was killed at 35 days after microinjection of AAV vectors. (A) Nissl-stained coronal brain section. (B) Fluorescent microscopic image showing EGFP expression, indicating the site of injection of AAV vectors. (C) In situ hybridization autoradiogram showing suppression of MC4R mRNA expression on the side of the PVN infected with AAV-MC4R-shRNA vectors. (D) Bright-field microscopic image showing no necrosis or abnormal cytoarchitecture in the PVN. CA1-3, area 1-3 Ammon's horn; CTX, cortex; DG, dentate gyrus; PVN, paraventricular nucleus of hypothalamus; STR, striatum; $\mathrm{TH}$, thalamus.

mRNA expression in the DMH, striatum, and amygdala was not affected (Fig. 8D-F). These data indicate that MC4R expression was specifically suppressed in the PVN of the rats, which was responsible for the observed changes in food intake and body weight gain shown above in Fig. 7 .

\section{Discussion}

Central melanocortin signaling is unique in that it is governed by dual circuits of the endogenous agonist $\alpha-\mathrm{MSH}$ and endogenous antagonist AgRP. Of the five melanocortin receptors identified to date, $\mathrm{MC} 4 \mathrm{R}$ is well established to exert physiological functions on feeding behavior and weight regulation. Increasing or decreasing activity of MC4R induces opposite effects on food intake and body weight gain (Huszar et al. 1997, Thiele et al. 1998, Marsh et al. 1999). The dual regulation of MC4R activity by endogenous ligands implies that information flow for executing feeding behavior and energy balance through MC4R signaling is tightly controlled. One important question that remains to be further addressed is what neuroanatomical and neurochemical substrates convey MC4R signaling in the control of energy homeostasis. Previous studies using the gene targeting strategy in MC4Rnull mice have narrowed down the neuronal circuit involving the PVN and amygdala that mediate MC4R function in the regulation of food intake but not energy expenditure (Balthasar et al. 2005). The present study examined the specific role of MC4R on PVN neurons in the regulation of food intake and body weight in the normally developed rat. The regionspecific MC4R knockdown in the PVN was achieved by intra-PVN injection of AAV-mediated shRNAs that specifically target MC4R. We found that MC4R knockdown in the PVN of the adult rat stimulates food intake and promote body weight gain in response to high-fat diet challenge.

The finding that the sequence-specific RNAi pathway can be artificially triggered in mammalian cells by introducing siRNAs or shRNAs led to rapid development of RNAi as an efficient tool for the study of gene function (Elbashir et al. 2001, Smart et al. 2005). Plasmid-based shRNA induces stable and constitutive production of siRNA in cells via Dicer-dependent cleavage. Dicer is the rate-limiting enzyme for shRNA processing into functional siRNAs for silencing of a target gene (Tijsterman \& Plasterk 2004). Overexpression of Dicer in Hela cells has been shown to enhance shRNA-mediated gene silencing by 30\% (Mikuma et al. 2004). Thus, the presence as well as the expression level of Dicer in the targeted brain region is a determining factor for achieving efficient gene silencing using shRNAs. Although Dicer had been identified in neurons (Lugli et al. 2005), its distribution in discrete regions of the 

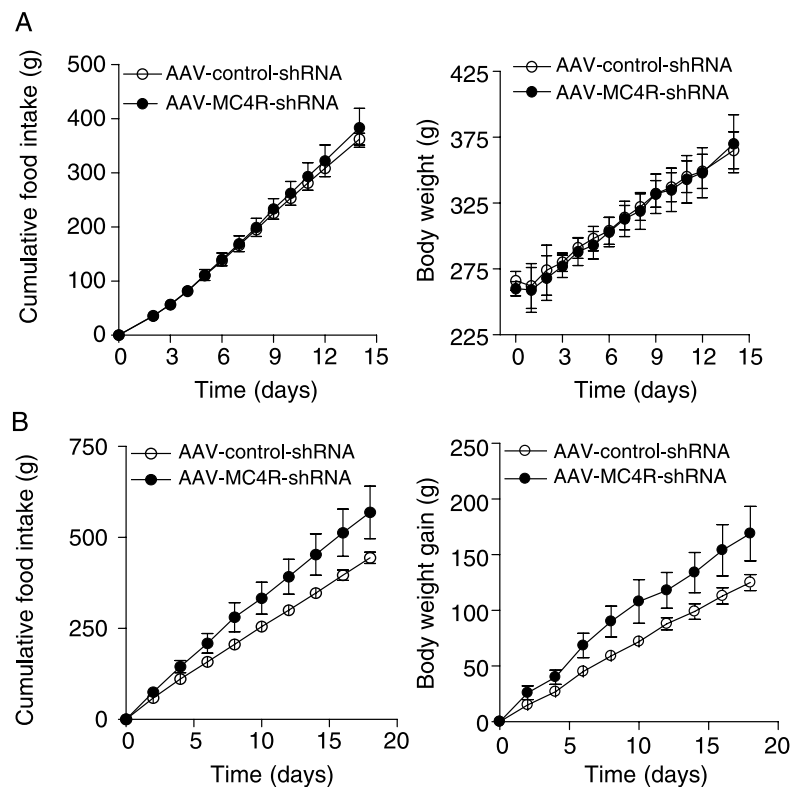

Figure 7 Effect of AAV-mediated MC4R knockdown in the PVN on food intake and body weight gain. AAV-MC4R-shRNA-EGFP or AAV-control-shRNA-EGFP was stereotaxically delivered into the PVN. After surgery, animals were fed a regular chow diet for 2 weeks, and then a high-fat diet for 3 weeks. (A) Accumulative food intake (left panel) and body weight (right panel) changes during the first 2 weeks post-injection in which a regular chow diet was provided to the rats. (B) Accumulative food intake (left panel) and body weight gain (right panel) measured for 3 weeks in response to a high-fat diet. AAV-control-shRNA-EGFP, $n=7$; AAV-MC4RshRNA-EGFP, $n=4$.

brain has not been revealed. Our in situ hybridization results show that Dicer expression is heterogeneous in the brain. Within the hypothalamus, the PVN expresses relatively high levels of Dicer mRNA. The abundance of Dicer in the PVN implies that exogenous shRNAs can be effectively processed into functional siRNAs in this brain region, indicative of the feasibility for AAV-shRNA-induced RNAi. However, the expression level of Dicer expression may not correlate with gene-silencing efficiency.

We employed AAV2 to deliver shRNAs into the PVN to achieve long-term expression of shRNAs and silence MC4R locally. One important feature of AAV2 is that this vector remains confined to the injection site and specifically infects neurons rather than glia (Bartlett et al. 1998, Tomar et al. 2003, Grimm et al. 2005). By inserting an additional expression cassette to encode EGFP for fluorescent detection, we were able to visualize the injection site and AAV transduction in the brain. We found that the EGFP-expressing cells were restricted to the deposition site. Microinjection of AAV vectors carrying MC4R shRNA into the PVN induced a significant reduction of MC4R mRNA expression in this region. This gene-silencing effect is specific to MC4R as aVP mRNA within the PVN was not affected. In addition, MC4R mRNA expression outside the PVN such as the DMH, amygdala, and striatum was not altered, suggesting that MC4R knockdown is not resultant from a generalized effect. These results support the efficacy and specificity of MC4R silencing in the PVN.

Whole-body deletion of the MC4R from the mouse genome results in severe adult obesity and hyperphagia (Huszar et al. 1997, Butler et al. 2001, Balthasar et al. 2005). Reactivation of MC4R in Sim1-expressing neurons, primarily in the PVN and amygdala, can normalize hyperphagia and reduce body weight gain without affecting energy expenditure in MC4R-null mice (Balthasar et al. 2005), suggesting that MC4R functions in appetite control are mediated by limited neuronal populations. Moreover, the same study has shown that specific reactivation of MC4R by injecting AAV-Cre into the PVN of adult loxP-modified, MC4R-null mice results in a mild decrease in body weight, but produces no significant effect on food intake (Balthasar et al. 2005). These results support an important role of MC4R on PVN neurons in energy homeostasis. However, one cannot rule out possible developmental maladaptation in this mouse model because of the lack of MC4R in other brain regions and tissues during development. In this study, we demonstrate that selective MC4R knockdown in PVN neurons of the adult wild-type rat results in no changes in food intake and body weight when animals are fed with a regular diet within 2 weeks, but causes increased feeding and excessive weight gain in response to a high-fat diet. It is unlikely that the unaltered feeding and body weight in response to the regular diet is due to insufficient gene suppression because AAV-mediated gene expression occurs within 3 days (Flotte et al. 1993, Takahashi et al. 2002) and AAV-mediated effective gene silencing has been reported at days 6-10 after injection of AAV-shRNA (Hommel et al. 2003, Wang et al. 2006, Paskowitz et al. 2007). Thus, our results suggest that the disruption of MC4R in PVN neurons increases the sensitivity to diet-induced hyperphagia and obesity. It is speculative that MC4R signaling in PVN neurons may normally protect against overeating and weight gain in response to dietary challenges. It is noted that the degree of increases in food intake and body weight induced by MC4R knockdown in the PVN is not as pronounced as the opposite effect of restoration of MC4R in Sim1-expressing neurons observed in MC4R-null mice (Balthasar et al. 2005). This could be due to incomplete suppression of MC4R expression in the PVN induced by $\mathrm{AAV}$ vectors in our study or the contribution of MC4R functions in Sim1 neurons located in areas other than the PVN. Another possibility could be the housing condition of the animals. In our studies, the rats were singly housed following stereotaxic injection in order to monitor food intake. It has been reported that MC4R-null mice that are individually housed and fed low-fat diets are not hyperphagic. However, these mice become hyperphagic after exposure to high-fat diets (Butler et al. 2001), suggesting the importance of the interplay between gene, diet, and environment in the development of obesity phenotypes.

The neurochemical substrates convey MC4R signaling in PVN neurons are currently unknown. A variety of neuronal cell populations with distinct neurochemical features have been 

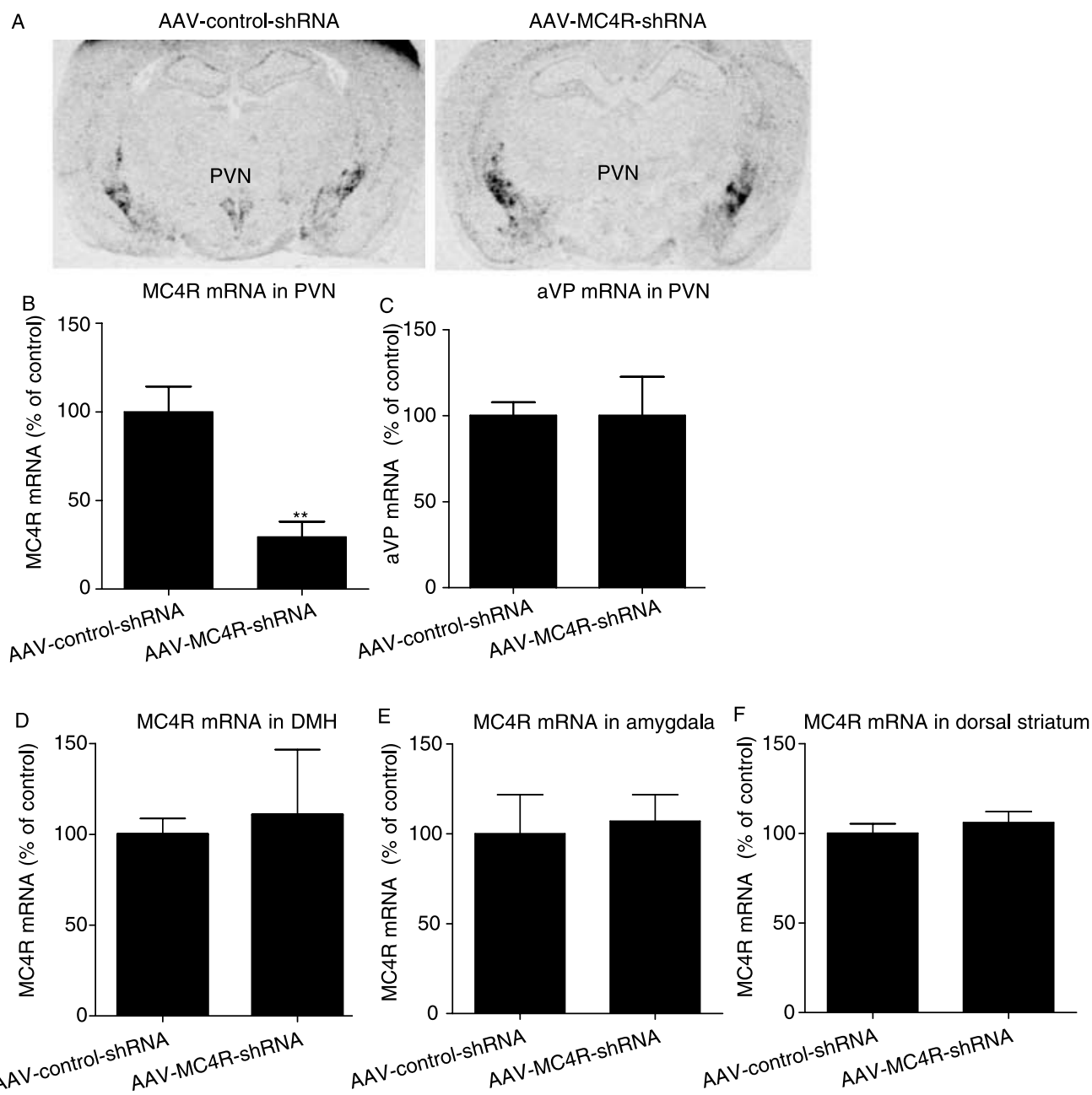

Figure 8 AAV-mediated MC4R knockdown in the PVN. (A) Representative images of in situ hybridization autoradiograms showing reduction of MC4R mRNA in the PVN by stereotaxic microinjection of AAV-MC4RshRNA-EGFP vectors in comparison with AAV-control-shRNA vectors 35 days after injection. Quantitative data indicate that (B) AAV-MC4R-shRNA-EGFP significantly reduces the level of MC4R mRNA expression in the PVN, but exhibits (C) no significant effects on aVP mRNA expression in the PVN. Also, the levels of MC4R mRNA expression in the (D) dorsal medial hypothalamus (DMH), (E) amgydala, and (F) dorsal striatum were not significantly affected. AAV-control-shRNA-EGFP, $n=5$; AAV-MC4R-shRNA-EGFP, $n=4$. ${ }^{* *} P<0 \cdot 01$.

revealed in the PVN. Of particular interest is corticotropinreleasing hormone $(\mathrm{CRH})$ neurons that express MC4R $(\mathrm{Lu}$ et al. 2003) and are innervated by POMC and AgRP neurons (Liposits et al. 1988, Fekete et al. 2000, Mihaly et al. 2002). It has been shown that CRH elicits anorectic actions when administered centrally (Rivest et al. 1989, Heinrichs \& Richard 1999, Richard et al. 2002), and food deprivation decreases CRH mRNA expression selectively in the PVN (Suemaru et al. 1986, Brady et al. 1990). Lesions of CRH neurons in the PVN markedly enhance feeding (Menzaghi et al. 1993). Activation of MC4R has been shown to stimulate $\mathrm{CRH}$ gene transcription in PVN neurons ( $\mathrm{Lu}$ et al. 2003). In addition, MC4R agonist-induced decrease in food intake can be attenuated by $\mathrm{CRH}$ receptor antagonists (Lu et al. 2003).
Lastly, MC4R-null mice respond to the inhibitory effects of CRH on feeding (Marsh et al. 1999), suggesting that CRH lies downstream of the MC4R signaling in the control of appetite. Another potential neuronal population in the PVN relevant to MC4R regulation of food intake is thyroid-releasing hormone (TRH) neurons. It has been shown that a large proportion of TRH neurons express MC4R (Liu et al. 2003) and receive input from POMC and AgRP neurons (Fekete et al. 2000). Moreover, food deprivation inhibits TRH mRNA expression in the PVN (Fekete et al. 2000). Central administration of $\mathrm{TRH}$ decreases food intake independent of its actions on thyroid function (Choi et al. 2002, Steward et al. 2003, Schuhler et al. 2007). Taken together, these findings suggest that $\mathrm{CRH}$ and $\mathrm{TRH}$ neurons may be effector neurons of 
MC4R signaling in the PVN in the regulation of energy homeostasis. Further investigation is required to test these possibilities.

\section{Acknowledgements}

This work was supported by American Heart Association Scientist Development Award AHA0530345N and National Institute of Health grants MH073844 and MH076929 (X Y L). The authors declare that there is no conflict of interest that would prejudice the impartiality of this scientific work.

\section{References}

Balthasar N, Dalgaard LT, Lee CE, Yu J, Funahashi H, Williams T, Ferreira M, Tang V, McGovern RA, Kenny CD et al. 2005 Divergence of melanocortin pathways in the control of food intake and energy expenditure. Cell 123 493-505.

Bartlett JS, Samulski RJ \& McCown TJ 1998 Selective and rapid uptake of adeno-associated virus type 2 in brain. Human Gene Therapy 9 1181-1186.

Brady LS, Smith MA, Gold PW \& Herkenham M 1990 Altered expression of hypothalamic neuropeptide mRNAs in food-restricted and food-deprived rats. Neuroendocrinology $\mathbf{5 2} 441-447$.

Butler AA, Marks DL, Fan W, Kuhn CM, Bartolome M \& Cone RD 2001 Melanocortin-4 receptor is required for acute homeostatic responses to increased dietary fat. Nature Neuroscience 4 605-611.

Choi YH, Hartzell D, Azain MJ \& Baile CA 2002 TRH decreases food intake and increases water intake and body temperature in rats. Physiology and Behavior 77 1-4.

Cowley MA, Pronchuk N, Fan W, Dinulescu DM, Colmers WF \& Cone RD 1999 Integration of NPY, AGRP, and melanocortin signals in the hypothalamic paraventricular nucleus: evidence of a cellular basis for the adipostat. Neuron 24 155-163.

Elbashir SM, Harborth J, Lendeckel W, Yalcin A, Weber K \& Tuschl T 2001 Duplexes of 21-nucleotide RNAs mediate RNA interference in cultured mammalian cells. Nature $\mathbf{4 1 1}$ 494-498.

Fekete C, Legradi G, Mihaly E, Huang QH, Tatro JB, Rand WM, Emerson CH \& Lechan RM 2000 alpha-Melanocyte-stimulating hormone is contained in nerve terminals innervating thyrotropin-releasing hormonesynthesizing neurons in the hypothalamic paraventricular nucleus and prevents fasting-induced suppression of prothyrotropin-releasing hormone gene expression. Journal of Neuroscience 20 1550-1558.

Flotte TR, Afione SA, Conrad C, McGrath SA, Solow R, Oka H, Zeitlin PL, Guggino WB \& Carter BJ 1993 Stable in vivo expression of the cystic fibrosis transmembrane conductance regulator with an adeno-associated virus vector. PNAS 90 10613-10617.

Grimm D, Pandey K \& Kay MA 2005 Adeno-associated virus vectors for short hairpin RNA expression. Methods in Enzymology 392 381-405.

Heinrichs SC \& Richard D 1999 The role of corticotropin-releasing factor and urocortin in the modulation of ingestive behavior. Neuropeptides 33 350-359.

Hommel JD, Sears RM, Georgescu D, Simmons DL \& DiLeone RJ 2003 Local gene knockdown in the brain using viral-mediated RNA interference. Nature Medicine 9 1539-1544.

Huszar D, Lynch CA, Fairchild-Huntress V, Dunmore JH, Fang Q, Berkemeier LR, Gu W, Kesterson RA, Boston BA, Cone RD et al. 1997 Targeted disruption of the melanocortin-4 receptor results in obesity in mice. Cell $\mathbf{8 8}$ 131-141.

Kas MJ, Tiesjema B, van Dijk G, Garner KM, Barsh GS, ter Brake O, Verhaagen J \& Adan RA 2004 Induction of brain-region-specific forms of obesity by agouti. Journal of Neuroscience 24 10176-10181.
Kask A, Schioth HB, Harro J, Wikberg JE \& Rago L 2000 Orexigenic effect of the melanocortin MC4 receptor antagonist HS014 is inhibited only partially by neuropeptide Y Y1 receptor selective antagonists. Canadian Journal of Physiology and Pharmacology 78 143-149.

Kim MS, Rossi M, Abusnana S, Sunter D, Morgan DG, Small CJ, Edwards CM, Heath MM, Stanley SA, Seal LJ et al. 2000 Hypothalamic localization of the feeding effect of agouti-related peptide and alpha-melanocyte-stimulating hormone. Diabetes 49 177-182.

Kishi T, Aschkenasi CJ, Lee CE, Mountjoy KG, Saper CB \& Elmquist JK 2003 Expression of melanocortin 4 receptor mRNA in the central nervous system of the rat. Journal of Comparative Neurology 457 213-235.

Liposits Z, Sievers L \& Paull WK 1988 Neuropeptide-Y and ACTHimmunoreactive innervation of corticotropin releasing factor (CRF)synthesizing neurons in the hypothalamus of the rat. An immunocytochemical analysis at the light and electron microscopic levels. Histochemistry 88 227-234.

Liu H, Kishi T, Roseberry AG, Cai X, Lee CE, Montez JM, Friedman JM \& Elmquist JK 2003 Transgenic mice expressing green fluorescent protein under the control of the melanocortin-4 receptor promoter. Journal of Neuroscience 23 7143-7154.

Lu XY, Shieh KR, Kabbaj M, Barsh GS, Akil H \& Watson SJ 2002 Diurnal rhythm of agouti-related protein and its relation to corticosterone and food intake. Endocrinology 143 3905-3915.

Lu XY, Barsh GS, Akil H \& Watson SJ 2003 Interaction between alphamelanocyte-stimulating hormone and corticotropin-releasing hormone in the regulation of feeding and hypothalamo-pituitary-adrenal responses. Journal of Neuroscience 23 7863-7872.

Lugli G, Larson J, Martone ME, Jones Y \& Smalheiser NR 2005 Dicer and eIF2c are enriched at postsynaptic densities in adult mouse brain and are modified by neuronal activity in a calpain-dependent manner. Journal of Neurochemistry 94 896-905.

Marsh DJ, Hollopeter G, Huszar D, Laufer R, Yagaloff KA, Fisher SL, Burn P \& Palmiter RD 1999 Response of melanocortin-4 receptor-deficient mice to anorectic and orexigenic peptides. Nature Genetics 21 119-122.

Menzaghi F, Heinrichs SC, Pich EM, Tilders FJ \& Koob GF 1993 Functional impairment of hypothalamic corticotropin-releasing factor neurons with immunotargeted toxins enhances food intake induced by neuropeptide $\mathrm{Y}$. Brain Research 618 76-82.

Mihaly E, Fekete C, Lechan RM \& Liposits Z 2002 Corticotropin-releasing hormone-synthesizing neurons of the human hypothalamus receive neuropeptide Y-immunoreactive innervation from neurons residing primarily outside the infundibular nucleus. Journal of Comparative Neurology $446235-243$.

Mikuma T, Kawasaki H, Yamamoto Y \& Taira K 2004 Overexpression of Dicer enhances RNAi-mediated gene silencing by short-hairpin RNAs (shRNAs) in human cells. Nucleic Acids Symposium Series 48 191-192.

Mountjoy KG, Mortrud MT, Low MJ, Simerly RB \& Cone RD 1994 Localization of the melanocortin-4 receptor (MC4-R) in neuroendocrine and autonomic control circuits in the brain. Molecular Endocrinology 8 1298-1308.

Paskowitz DM, Greenberg KP, Yasumura D, Grimm D, Yang H, Duncan JL, Kay MA, Lavail MM, Flannery JG \& Vollrath D 2007 Rapid and stable knockdown of an endogenous gene in retinal pigment epithelium. Human Gene Therapy 18 871-880.

Paxinos G \& Watson C 1998 The Rat Brain in Stereotaxic Coordinates. 4 edn, San Diego: Academic Press.

Paz J, Yao H, Lim HS, Lu XY \& Zhang W 2007 The neuroprotective role of attractin in neurodegeneration. Neurobiology of Aging 28 1446-1456.

Richard D, Lin Q \& Timofeeva E 2002 The corticotropin-releasing factor family of peptides and CRF receptors: their roles in the regulation of energy balance. European Journal of Pharmacology 440 189-197.

Rivest S, Deshaies Y \& Richard D 1989 Effects of corticotropin-releasing factor on energy balance in rats are sex dependent. American Journal of Physiology 257 R1417-R1422.

Sawchenko PE 1988 Effects of catecholamine-depleting medullary knife cuts on corticotropin-releasing factor and vasopressin immunoreactivity in the hypothalamus of normal and steroid-manipulated rats. Neuroendocrinology $\mathbf{4 8}$ 459-470.

Sawchenko PE 1998 Toward a new neurobiology of energy balance, appetite, and obesity: the anatomists weigh in. Journal of Comparative Neurology 402 435-441. 
Sawchenko PE \& Swanson LW 1983 The organization of forebrain afferents to the paraventricular and supraoptic nuclei of the rat. Journal of Comparative Neurology 218 121-144.

Schuhler S, Warner A, Finney N, Bennett GW, Ebling FJ \& Brameld JM 2007 Thyrotrophin-releasing hormone decreases feeding and increases body temperature, activity and oxygen consumption in Siberian hamsters. Journal of Neuroendocrinology 19 239-249.

Shioda S, Nakai Y, Iwase M \& Homma I 1990 Electron microscopic studies of medullary synaptic inputs to vasopressin-containing neurons in the hypothalamic paraventricular nucleus. Journal of Electron Microscopy 39 501-507.

Smart N, Scambler PJ \& Riley PR 2005 A rapid and sensitive assay for quantification of siRNA efficiency and specificity. Biological Procedures Online $71-7$.

Steward CA, Horan TL, Schuhler S, Bennett GW \& Ebling FJ 2003 Central administration of thyrotropin releasing hormone $(\mathrm{TRH})$ and related peptides inhibits feeding behavior in the Siberian hamster. Neuroreport 14 687-691.

Suemaru S, Hashimoto K, Hattori T, Inoue H, Kageyama J \& Ota Z 1986 Starvation-induced changes in rat brain corticotropin-releasing factor (CRF) and pituitary-adrenocortical response. Life Science 39 1161-1166.

Takahashi H, Hirai Y, Migita M, Seino Y, Fukuda Y, Sakuraba H, Kase R, Kobayashi T, Hashimoto Y \& Shimada T 2002 Long-term systemic therapy of Fabry disease in a knockout mouse by adeno-associated virus-mediated muscle-directed gene transfer. PNAS 99 13777-13782.

Thiele TE, van Dijk G, Yagaloff KA, Fisher SL, Schwartz M, Burn P \& Seeley RJ 1998 Central infusion of melanocortin agonist MTII in rats: assessment of c-Fos expression and taste aversion. American Journal of Physiology 274 R248-R254.

Tijsterman M \& Plasterk RH 2004 Dicers at RISC; the mechanism of RNAi. Cell 117 1-3.
Tomar RS, Matta H \& Chaudhary PM 2003 Use of adeno-associated viral vector for delivery of small interfering RNA. Oncogene 22 5712-5715.

Vaisse C, Clement K, Durand E, Hercberg S, Guy-Grand B \& Froguel P 2000 Melanocortin-4 receptor mutations are a frequent and heterogeneous cause of morbid obesity. Journal of Clinical Investigation 106 253-262.

Wang X, Skelley L, Cade R \& Sun Z 2006 AAV delivery of mineralocorticoid receptor shRNA prevents progression of cold-induced hypertension and attenuates renal damage. Gene Therapy 13 1097-1103.

Weingarten HP, Chang PK \& McDonald TJ 1985 Comparison of the metabolic and behavioral disturbances following paraventricular- and ventromedialhypothalamic lesions. Brain Research Bulletin 14 551-559.

Wirth MM \& Giraudo SQ 2001 Effect of Agouti-related protein delivered to the dorsomedial nucleus of the hypothalamus on intake of a preferred versus a non-preferred diet. Brain Research 897 169-174.

Xie Q, Hare J, Turnigan J \& Chapman MS 2004 Large-scale production, purification and crystallization of wild-type adeno-associated virus- 2 . Journal of Virological Methods 122 17-27.

Yeo GS, Farooqi IS, Aminian S, Halsall DJ, Stanhope RG \& O'Rahilly S 1998 A frameshift mutation in MC4R associated with dominantly inherited human obesity. Nature Genetics 20 111-112.

Received in final form 20 March 2008

Accepted 26 March 2006

Made available online as an Accepted Preprint

27 March 2008 